\title{
Midline Suboccipital Subtonsillar Approach with C1 Laminectomy for Resection of Foramen Magnum Meningioma: 2-Dimensional Operative Video
}

\author{
Stefan Lieber ${ }^{1,2}$ Maximiliano Nunez ${ }^{2}$ Rocio Evangelista-Zamora ${ }^{1}$ Marcos Tatagiba ${ }^{1}$ \\ ${ }^{1}$ Department of Neurological Surgery, Eberhard-Karls-University, \\ University Hospital, Tübingen, Germany \\ 2 Department of Neurological Surgery, Microsurgical Neuroanatomy Lab, \\ University of Pittsburgh, Pittsburgh, Pennsylvania, United States \\ Address for correspondence Stefan Lieber, MD, Department of \\ Neurological Surgery, Eberhard-Karls-University, \\ Hoppe-Seyler-Strasse 3, Tübingen, D-72076, Germany \\ (e-mail: Stefan.Lieber@uclmail.net).
}

J Neurol Surg B 2019;80(suppl S4):S365-S367.

We present a case of a medium-sized foramen magnum meningioma that was resected through a midline suboccipital subtonsillar approach with C1 laminectomy in prone (Concorde) position. The patient is a 77-year-old woman with a 6-month history of intermittent vertigo, moderate gait instability, and slight decline of memory.

On magnetic resonance imaging (MRI) an extra-axial intradural lesion was discovered that originated from the right epicondylar region just inferior to the jugular tubercle and occupied the anterolateral aspect of the foramen magnum. There was moderate transposition and compression of the medulla at the level of the cerebellar tonsils. On physical examination the patient was ambulatory independently without motor weakness but exhibited some gait instability. The function of the lower cranial nerves was preserved.

A gross-total resection was achieved, histopathology confirmed a WHO grade-I meningothelial meningioma with a low-proliferation index. The patient was discharged home 5 days after surgery, her gait instability improved significantly immediately after surgery and had resolved completely after 2 weeks of inpatient rehabilitation. There was no other neurological deficit. At 3-month follow-up MRI, there was no indication of meningioma residual or recurrence.

In summary, the midline suboccipital subtonsillar approach is a powerful tool with limited morbidity in the armamentarium for the microsurgical management of a variety of pathologies residing in the posterior cranial fossa and the craniocervical junction. Oftentimes the space created by the pathology opens up corridors that can be exploited for microsurgical access to avoid more extensive surgical approaches. The link to the video can be found at: https://youtu.be/0uUxs13ze7w.

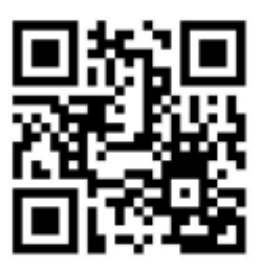

received

May 19, 2019 accepted after revision August 24, 2019 published online October 28, 2019 www.thieme.com/skullbasevideos

www.thieme.com/jnlsbvideos
(C) 2019 Georg Thieme Verlag KG Stuttgart · New York

License terms

10.1055/s-0039-1698823. ISSN 2193-6331.

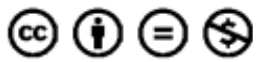



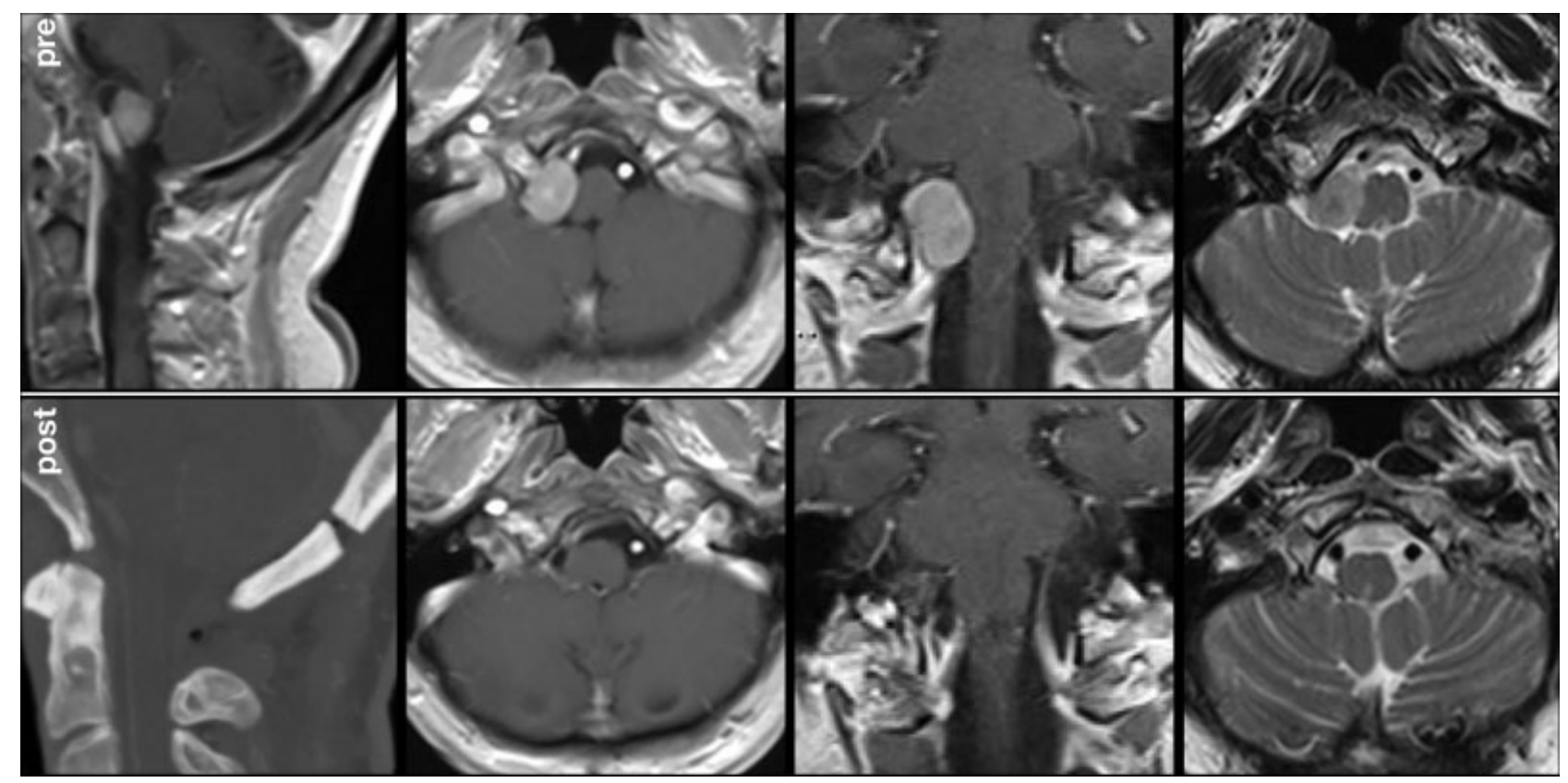

Fig. 1 Preoperative MRI and postoperative CT and MRI studies. The meningioma originated from the right epicondylar region just inferior to the jugular tubercle and occupied the anterolateral aspect of the foramen magnum. CT, computed tomography; MRI, magnetic resonance imaging .

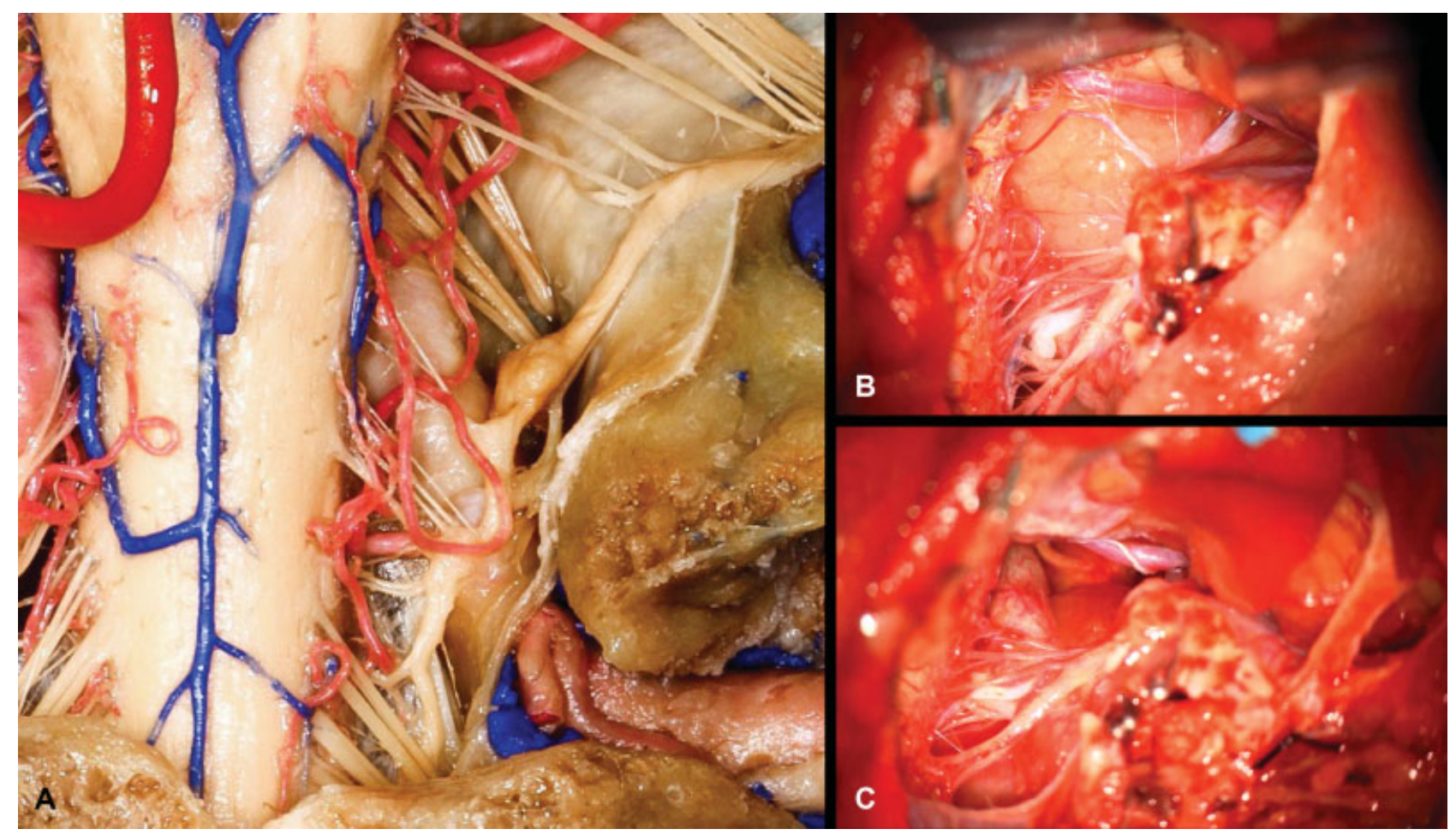

Fig. 2 (A) anatomical dissection (fixed, silicone-injected human cadaver), depicting a right-sided craniocervical junction from the level of the jugular foramen to the posterior arch of C1. (B and C) operative still images of the suboccipital subtonsillar approach; (B) the meningioma occupies the anterolateral aspect of the foramen magnum on the right side. Hypoglossal nerve and vertebral artery (V4 segment) are being transposed anteriorly, the posterior inferior cerebellar artery and fascicles of the accessory nerve are being transposed posteriorly. (C) After complete microsurgical excision and bipolar coagulation of the dural attachment sites, the hypoglossal nerve and the vertebral artery can be seen in the depth of the surgical field. 


\section{Publication Comments}

In this video, the authors describe the use of the midline sub occipital subtonsillar approach with c1 laminectomy for the resection of a foramen magnum meningioma in a symptomatic 77-year-old patient. This video highlights the importance of this approach in the armamentarium of a skull base surgeon as well it enhances the close relationship of these tumors with critical neurovascular structures. I congratulate the authors for the surgical result and the surgeon's mastery.

Diego Mendez Rosito, MD CMN 20 de Noviembre Mexico City, Mexico
Conflict of Interest

None declared. 